\section{Convexity Properties of Detection Probability for Noncoherent Detection of a Modulated Sinusoidal Carrier}

\author{
Cuneyd Ozturk ${ }^{\mathbb{D}}$, Berkan Dulek ${ }^{\mathbb{D}}$, Member, IEEE, \\ and Sinan Gezici ${ }^{\mathbb{D}}$, Senior Member, IEEE
}

\begin{abstract}
In this correspondence paper, the problem of noncoherent detection of a sinusoidal carrier is considered in the presence of Gaussian noise. The convexity properties of the detection probability are characterized with respect to the signal-to-noise ratio (SNR). It is proved that the detection probability is a strictly concave function of SNR when the false alarm probability $\alpha$ satisfies $\alpha>e^{-2}$, and it is first a strictly convex function and then a strictly concave function of SNR for $\alpha<e^{-2}$. In addition, optimal power allocation strategies are derived under average and peak power constraints. It is shown that $\mathrm{ON}-\mathrm{OFF}$ signaling can be optimal for $\alpha<e^{-2}$ depending on the power constraints, whereas transmission at a constant power level that is equal to the average power limit is optimal in all other cases.
\end{abstract}

Index Terms-Detection, Neyman-Pearson, noncoherent, probability of detection, convexity, power allocation.

\section{INTRODUCTION}

Noncoherent detection is employed in various wireless applications due to its practicality and low complexity [1], [2]. In the noncoherent detection framework, the receiver does not exploit the phase information of the carrier, which modulates the message signal. In this paper, the problem of noncoherent detection of a modulated sinusoidal carrier is considered [2, pp. 65-72]. In this problem, the detection probability can explicitly be obtained in terms of the false alarm probability and signal-to-noise ratio (SNR). The aim in this paper is to investigate the convexity properties of the detection probability with respect to SNR and consequently to develop optimal power allocation strategies for noncoherent detection of a modulated sinusoidal carrier.

Convexity properties of error probability and detection probability are analyzed in various studies in the literature, such as [3]-[5]. The work in [3] investigates the convexity properties of the error probability corresponding to the maximum likelihood (ML) detector for a binary hypothesis-testing problem. The theoretical analysis reveals that the error probability of the ML detector is convex with respect to the signal

Manuscript received May 24, 2018; revised August 16, 2018 and October 5 , 2018; accepted October 6, 2018. Date of publication October 17, 2018; date of current version December 14, 2018. The review of this paper was coordinated by Prof. D. B. da Costa. (Corresponding author: Sinan Gezici.)

C. Ozturk and S. Gezici are with the Department of Electrical and Electronics Engineering, Bilkent University, Ankara 06800, Turkey (e-mail: cuneyd@ ee.bilkent.edu.tr; gezici@ee.bilkent.edu.tr).

B. Dulek is with the Department of Electrical and Electronics Engineering, Hacettepe University, Ankara 06800, Turkey (e-mail: berkan@ ee.hacettepe.edu.tr)

Color versions of one or more of the figures in this paper are available online at http://ieeexplore.ieee.org.

Digital Object Identifier 10.1109/TVT.2018.2876516 power when the noise has a unimodal distribution [3]. The results in [3] are extended to the multi-dimensional case in [4] by employing the ML detector for additive white Gaussian noise (AWGN) channels with flat and non-flat fading. It is shown that when the dimension of the constellation is less than or equal to two, the symbol error rate is always convex in SNR. On the other hand, when the dimension is larger than two, the symbol error rate is concave at low SNRs and convex at high SNRs [4]. In [5], the convexity properties of the detection probability are investigated in the Neyman-Pearson (NP) framework. It is proved that the detection probability is strictly concave in SNR when the false alarm probability $\alpha$ satisfies $\alpha \geq Q(2)$ and has two inflection points when $\alpha<Q(2)$, where $Q(\cdot)$ denotes the $Q$-function[5]. Based on this result, the optimal power allocation strategy is proposed for $\alpha<Q(2)$, which can significantly improve the detection probability in some cases via time sharing between different power levels.

In this paper, we consider the noncoherent detection problem for a modulated sinusoidal carrier within the NP framework [2, pp. 65-72]. The main contribution of this paper is to characterize the convexity properties of the detection probability with respect to SNR for all levels of false alarm probability, which is not available in the literature. We prove that the detection probability is strictly concave in SNR when the false alarm probability satisfies $\alpha>e^{-2}$, and starts as a strictly convex function and continues as a strictly concave function of SNR for $\alpha<e^{-2}$. Due to the existence of the convex region for $\alpha<e^{-2}$, the detection probability performance can be improved via time sharing between different power levels, which is analyzed by characterizing the optimal power allocation under average and peak power constraints. It is shown that, for $\alpha<e^{-2}$, onoff signaling can facilitate significant improvements in the detection performance when the average power constraint is less than a fixed value.

\section{SYSTEM MODEL}

Consider the problem of noncoherent detection of a sinusoidal carrier in the presence of Gaussian noise. Namely, the aim is to decide between two hypotheses $\mathcal{H}_{0}$ versus $\mathcal{H}_{1}$ based on a vector-valued observation $\boldsymbol{Y}=\left[Y_{1}, \ldots, Y_{n}\right]^{T}$, which is described as follows:

$$
\mathcal{H}_{0}: Y_{k}=N_{k}, \mathcal{H}_{1}: Y_{k}=\sqrt{P} s_{k}(\theta)+N_{k}, \text { for } k=1, \ldots, n
$$

where the noise components $N_{k}$ are zero-mean independent and identically distributed (i.i.d.) Gaussian random variables with variance $\sigma^{2}$ for $k=1, \ldots, n$, parameter $P$ determines the power of the transmitted signal, and $s(\theta)=\left[s_{1}(\theta), \ldots, s_{n}(\theta)\right]^{T}$ is a vector-valued function of $\theta$, with $s_{k}(\theta)$ 's being samples from a modulated sinusoidal carrier as follows [2, p. 65]:

$$
s_{k}(\theta)=a_{k} \sin \left((k-1) \omega_{c} T_{s}+\theta\right) \text { for } k=1, \ldots, n
$$

In (2), $w_{c}$ is the carrier (angular) frequency, $T_{s}$ is the sampling interval, $a_{1}, \ldots, a_{n}$ are samples of bandlimited waveform $a(t)$ which modulates the sinusoidal carrier, and $\theta$ is the unknown phase of the carrier, which is 
modeled by a uniform random variable over $[0,2 \pi)$ that is independent of the noise components. It is assumed that $n \omega_{c} T_{s}=2 \pi m$ for some integer $m$, and $n / m$ (i.e., the number of samples taken per cycle of the sinusoid) is an integer larger than one [2].

Averaging over the uniform distribution of the phase $\theta$ and assuming that $a_{1}^{2}, \ldots, a_{n}^{2}$ vary slowly compared to twice the carrier frequency, the likelihood ratio for the problem specified by (1) and (2) can be expressed as

$$
L(\boldsymbol{y})=e^{-\frac{n \overline{a^{2}} P}{4 \sigma^{2}}} I_{0}\left(r P / \sigma^{2}\right),
$$

where $\overline{a^{2}}=\frac{1}{n} \sum_{k=1}^{n} a_{k}^{2}, I_{0}(\cdot)$ is the zeroth order modified Bessel function of the first kind, i.e. $I_{0}(x)=(1 / 2 \pi) \int_{0}^{2 \pi} e^{x \cos \theta} d \theta$ and $r=\sqrt{y_{c}^{2}+y_{s}^{2}}$, with $y_{c}=\sum_{k=1}^{n} a_{k} y_{k} \cos \left((k-1) \omega_{c} T_{s}\right)$ and $y_{s}=$ $\sum_{k=1}^{n} a_{k} y_{k} \sin \left((k-1) \omega_{c} T_{s}\right)$. From (3) and the monotonicity of $I_{0}(\cdot)$, the optimum likelihood ratio test can be implemented by comparing $r$ against a threshold. Then, the optimum size- $\alpha$ NP decision rule can be specified as [2, p. 70]

$$
r \underset{\mathcal{H}_{0}}{\stackrel{\mathcal{H}_{1}}{\gtrless}}\left(n \sigma^{2} \overline{a^{2}} \log (1 / \alpha)\right)^{1 / 2}
$$

Let $\gamma \triangleq n \overline{a^{2}} P /\left(2 \sigma^{2}\right)$ represent the SNR. The decision rule in (4) achieves the following probability of detection:

$$
P_{d}(\gamma, \alpha)=Q_{1}[\sqrt{\gamma}, \sqrt{-2 \log \alpha}]
$$

where $\alpha$ is the false alarm probability and $Q_{1}[y, b]$ is Marcum's $Q$-function of order 1 , which is given by $Q_{1}[y, b]=$ $\int_{b}^{\infty} t e^{-\left(t^{2}+y^{2}\right) / 2} I_{0}(t y) d t[2]$.

\section{Convexity Properties In Signal Power AND OPTIMAL POWER ALLOCATION}

In this section, the aim is to analyze the convexity properties of the detection probability in (5) with respect to SNR (or, equivalently signal power), and subsequently to develop optimal power allocation strategies that achieve the maximum average detection probability under average and peak power constraints.

\section{A. Convexity/Concavity Results}

We start with analyzing the convexity of $Q_{1}[\sqrt{\gamma}, \sqrt{-2 \log \alpha}]$ in (5) with respect to $\gamma$. To simplify the notation, the following definition is employed: $f(\alpha) \triangleq \sqrt{-2 \log \alpha}$. Then, (5) becomes $P_{d}(\gamma, \alpha)=$ $Q_{1}[\sqrt{\gamma}, f(\alpha)]$.

Before analyzing the convexity of $P_{d}(\gamma, \alpha)$, it is recalled from [6, Thm. 1] that $P_{d}(\gamma, \alpha)$ is monotone increasing with respect to $\gamma$. Then, the following proposition characterizes the behavior of $P_{d}(\gamma, \alpha)$ for $\alpha>e^{-2}$.

Proposition 1: If the false alarm probability satisfies $\alpha>e^{-2}$, then $P_{d}(\gamma, \alpha)$ is a strictly concave and monotonically increasing function of $\gamma$ for all $\gamma \in[0, \infty)$.
Proof: From [7, Eq. (16)], the second derivative of $Q_{1}[\sqrt{\gamma}, f(\alpha)]$ with respect to $\gamma$ can be expressed as

$$
\begin{aligned}
\frac{d^{2}}{d \gamma^{2}} Q_{1}[\sqrt{\gamma}, f(\alpha)]= & (-2)^{-2} \sum_{p=0}^{2}(-1)^{p}\left(\begin{array}{l}
2 \\
p
\end{array}\right) Q_{1+p}[\sqrt{\gamma}, f(\alpha)] \\
= & \frac{1}{4}\left(Q_{1}[\sqrt{\gamma}, f(\alpha)]-2 Q_{2}[\sqrt{\gamma}, f(\alpha)]\right. \\
& \left.+Q_{3}[\sqrt{\gamma}, f(\alpha)]\right)
\end{aligned}
$$

where $Q_{i}[\cdot, \cdot]$ denotes Marcum's $Q$-function of order $i$. Then, via the recurrence relation of Marcum's $Q$-function in [7, Eq. (2)], (6) can be written as:

$$
\begin{aligned}
\frac{d^{2}}{d \gamma^{2}} Q_{1}[\sqrt{\gamma}, f(\alpha)]= & \frac{1}{4} \frac{f(\alpha)}{\sqrt{\gamma}} e^{-\frac{\gamma+(f(\alpha))^{2}}{2}} \\
& \times\left(\frac{f(\alpha)}{\sqrt{\gamma}} I_{2}(\sqrt{\gamma} f(\alpha))-I_{1}(\sqrt{\gamma} f(\alpha))\right),
\end{aligned}
$$

where $I_{i}(\cdot)$ denotes the $i$ th order modified Bessel function of the first kind. To prove the concavity, it is sufficient to consider the sign of $\left(\frac{f(\alpha)}{\sqrt{\gamma}} I_{2}(\sqrt{\gamma} f(\alpha))-I_{1}(\sqrt{\gamma} f(\alpha))\right)$ as the other terms are positive in (7). From the inequality given in [8, Eq. (2.21)], it is known that

$$
I_{2}(\sqrt{\gamma} f(\alpha))<I_{1}(\sqrt{\gamma} f(\alpha)) \frac{\sqrt{\gamma} f(\alpha)}{4} .
$$

Therefore, it follows that

$$
\frac{f(\alpha)}{\sqrt{\gamma}} I_{2}(\sqrt{\gamma} f(\alpha))-I_{1}(\sqrt{\gamma} f(\alpha))<\left(\frac{f(\alpha)^{2}}{4}-1\right) I_{1}(\sqrt{\gamma} f(\alpha))
$$

From (9), it is noted that if $f(\alpha)^{2}<4$ (equivalently, if $\alpha>e^{-2}$ ),

$$
\frac{f(\alpha)}{\sqrt{\gamma}} I_{2}(\sqrt{\gamma} f(\alpha))-I_{1}(\sqrt{\gamma} f(\alpha))<0
$$

is obtained, which concludes the proof.

Next, to investigate the convexity properties of $P_{d}(\gamma, \alpha)$ for $\alpha<$ $e^{-2}$, the following lemmas are presented, which are proved in the Appendix.

Lemma 1: If $\alpha<e^{-2}$, there exists $\hat{\gamma}>0$ such that the second derivative of $P_{d}(\gamma, \alpha)$ with respect to $\gamma$ is positive for $\gamma \in[0, \hat{\gamma}]$.

Lemma 2: If $\alpha<e^{-2}$, there exists $\tilde{\gamma}$ such that the second derivative of $P_{d}(\gamma, \alpha)$ with respect to $\gamma$ is negative for all $\gamma \geq \tilde{\gamma}$

Lemma 3: For $\alpha<e^{-2}$, there exists a unique inflection point $\gamma^{*}$ such that $P_{d}^{\prime \prime}\left(\gamma^{*}, \alpha\right)=0$, where $P_{d}^{\prime \prime}\left(\gamma^{*}, \alpha\right)$ denotes the second derivative of $P_{d}(\gamma, \alpha)$ with respect to $\gamma$ evaluated at $\gamma^{*}$.

Based on Lemma 1, Lemma 2, and Lemma 3, the convexity properties of $P_{d}(\gamma, \alpha)$ are characterized in the following proposition when the false alarm probability satisfies $\alpha<e^{-2}$.

Proposition 2: For $\alpha<e^{-2}$, there exists $\gamma_{\alpha}>0$ such that $P_{d}(\gamma, \alpha)$ is a strictly convex and monotonically increasing function of $\gamma$ in $\left[0, \gamma_{\alpha}\right)$ and a strictly concave and monotonically increasing function of $\gamma$ in $\left[\gamma_{\alpha}, \infty\right)$.

Proof: The proof follows from [9, Thm. 1], Lemma 1, Lemma 2, Lemma 3, and the Intermediate Value Theorem. 
Proposition 1 together with Proposition 2 characterize the convexity properties of the detection probability for all possible values of the false alarm probability $\alpha .^{1}$

\section{B. Optimal Power Allocation}

In this section, enhancement of detection performance via time sharing among different power levels is investigated. Consider a general time sharing strategy with time sharing factors $\left\{\lambda_{i}\right\}_{i=1}^{M}$ and corresponding SNR values $\left\{\gamma_{i}\right\}_{i=1}^{M}$, where $M$ denotes the number of SNR levels that can be employed during the time sharing operation, and $\lambda_{i}$ 's are nonnegative and sum to one. Then, the aim is to obtain the optimal strategy that maximizes the average detection probability under average and peak SNR (equivalently, power) constraints. Mathematically stated,

$$
\begin{array}{ll}
\max _{\left\{\lambda_{i}, \gamma_{i}\right\}_{i=1}^{M}} & \sum_{i=1}^{M} \lambda_{i} P_{d}\left(\gamma_{i}, \alpha\right) \\
\text { subject to } & \sum_{i=1}^{M} \lambda_{i} \gamma_{i} \leq \Gamma_{\text {avg }}, \sum_{i=1}^{M} \lambda_{i}=1 \\
& 0 \leq \gamma_{i} \leq \Gamma_{\text {peak }}, \lambda_{i} \geq 0 \quad i=1, \ldots, M
\end{array}
$$

where $\Gamma_{\text {avg }} \leq \Gamma_{\text {peak }}$ is assumed.

Since the detection probability is a monotonically increasing function of $\gamma$, the solution of (11) always operates at the average SNR limit $\Gamma_{\mathrm{avg}}$. In addition, for $\alpha>e^{-2}$, based on the strict concavity of the detection probability with respect to SNR (Proposition 1), it can be deduced that the solution of (11) is given by $\lambda_{k}^{*}=1, \lambda_{i}^{*}=0$ for $i \in\{1, \ldots, M\} \backslash\{k\}$ and $\gamma_{k}^{*}=\Gamma_{\text {avg }}$ for any $k \in\{1, \ldots, M\}$. In other words, when $\alpha>e^{-2}$, time sharing is not employed, and a constant transmission power that corresponds to the average SNR limit, $\Gamma_{\text {avg }}$, is used all the time.

On the other hand, for $\alpha<e^{-2}$, there exists an interval over which the detection probability is convex (Proposition 2). Hence, improvements in detection probability can be achieved via time sharing under certain scenarios. To characterize the optimal time sharing strategy (i.e., the solution of (11)) for $\alpha<e^{-2}$, the following lemma is presented first, which is proved in the Appendix.

Lemma 4: Let $\gamma_{\alpha}$ be the unique inflection point of $P_{d}(\gamma, \alpha)$ for $\alpha<e^{-2}$. Then, there exists $\gamma_{t}>\gamma_{\alpha}$ such that the line passing through points $\left(0, P_{d}(0, \alpha)\right)$ and $\left(\gamma_{t}, P_{d}\left(\gamma_{t}, \alpha\right)\right)$ is tangent to $P_{d}(\gamma, \alpha)$ at $\gamma_{t}$, and lies above $P_{d}(\gamma, \alpha)$ for all $\gamma>0$.

Based on Lemma 4, the optimal time sharing strategy for $\alpha<e^{-2}$ can be described as follows:

Proposition 3: Let $\alpha<e^{-2}$ and $\gamma_{t}$ be the tangent point defined as in Lemma 4.

i) If $\gamma_{t} \leq \Gamma_{\mathrm{avg}}$, the optimal strategy is to employ $\Gamma_{\mathrm{avg}}$ all the time.

ii) If $\Gamma_{\text {peak }} \geq \gamma_{t}>\Gamma_{\text {avg }}$, the optimal strategy is to time share between SNRs of 0 and $\gamma_{t}$, with fraction of time $\Gamma_{\mathrm{avg}} / \gamma_{t}$ allocated to the SNR of $\gamma_{t}{ }^{2}$

\footnotetext{
${ }^{1}$ It is worth mentioning that inflection point $\gamma_{\alpha}$ can easily be computed via a bisection search [5] since it is a root of the following equation: $v_{1}\left(\gamma_{\alpha} f(\alpha)\right)=$ $(f(\alpha))^{2}$, as shown in the proof of Lemma 3 .

${ }^{2}$ In practice, time sharing between different SNR values can be implemented by time sharing between different transmitter powers, controlled by the parameter $P$ in (1).
}

iii) If $\gamma_{t}>\Gamma_{\text {peak }}$, the optimal strategy is to time share between SNRs of 0 and $\Gamma_{\text {peak }}$, with fraction of time $\Gamma_{\text {avg }} / \Gamma_{\text {peak }}$ allocated to the SNR of $\Gamma_{\text {peak }}$.

Proof: Let the average SNR in (11b) and the average detection probability (objective function) in (11a) be denoted by $\sum_{i=1}^{M} \lambda_{i} \gamma_{i} \triangleq$ $\bar{\gamma}$ and $\sum_{i=1}^{M} \lambda_{i} P_{d}\left(\gamma_{i}, \alpha\right) \triangleq \bar{P}_{d}(\bar{\gamma}, \alpha)$, respectively. Consider $(i)$ and (ii), where $\gamma_{t} \leq \Gamma_{\text {peak }}$. Let $\bar{\gamma}$ be an average SNR. Then, according to the proposed strategy, the following average detection probability is achieved:

$$
\bar{P}_{d}^{*}(\bar{\gamma}, \alpha)= \begin{cases}P_{d}(\bar{\gamma}, \alpha), & \text { if } \bar{\gamma} \in\left(\gamma_{t}, \Gamma_{\text {peak }}\right] \\ P_{d}(0, \alpha)+\lambda \bar{\gamma}, & \text { if } \bar{\gamma} \in\left[0, \gamma_{t}\right]\end{cases}
$$

where $\lambda=\left(P_{d}\left(\gamma_{t}, \alpha\right)-P_{d}(0, \alpha)\right) / \gamma_{t}$. Since the aim in (11) is to maximize the average detection probability via time sharing, it can be shown that the optimal solution resides on the upper boundary of the convex hull of the $\gamma$ versus $P_{d}(\gamma, \alpha)$ curve for $\gamma \in\left[0, \Gamma_{\text {peak }}\right]$ (see, e.g., [10] for a similar scenario). Therefore, the proposition can be proved by showing that $\bar{P}_{d}^{*}(\gamma, \alpha)$ in (12) is the smallest concave function which is greater than or equal to $P_{d}(\gamma, \alpha)$; i.e., $\bar{P}_{d}^{*}(\gamma, \alpha)$ forms the upper boundary of the convex hull. First, it is clear that $\bar{P}_{d}^{*}(\gamma, \alpha)$ is a concave function of $\gamma$. Hence, for $\gamma>\gamma_{t}, \bar{P}_{d}^{*}(\gamma, \alpha)$ in (12) becomes the upper boundary of the convex hull by definition. For $\gamma \in\left[0, \gamma_{t}\right]$, suppose, towards a contradiction, that $\bar{P}_{d}^{*}(\gamma, \alpha)$ is not the smallest concave function greater than or equal to $P_{d}(\gamma, \alpha)$. This implies that there exists another function $g_{1}(\gamma, \alpha)$ which is concave and greater than or equal to $P_{d}(\gamma, \alpha)$, and that there exists $x \in\left[0, \gamma_{t}\right]$ such that $g_{1}(x, \alpha)<$ $\bar{P}_{d}^{*}(x, \alpha)$. As $x \in\left[0, \gamma_{t}\right]$, there exists $0<\beta<1$ such that $x=\beta \gamma_{t}$. Then, by the concavity of $g_{1}$, it is clear that $g_{1}(x, \alpha) \geq \beta g_{1}\left(\gamma_{t}, \alpha\right)+$ $(1-\beta) g_{1}(0, \alpha)$. Since $g_{1}$ is greater than or equal to $P_{d}(\gamma, \alpha)$, it is concluded that $g_{1}(x, \alpha) \geq \beta g_{1}\left(\gamma_{t}, \alpha\right)+(1-\beta) g_{1}(0, \alpha) \geq$ $\beta P_{d}\left(\gamma_{t}, \alpha\right)+(1-\beta) P_{d}(0, \alpha)=\bar{P}_{d}^{*}(x, \alpha)$, which contradicts the assumption of $g_{1}(x, \alpha)<\bar{P}_{d}^{*}(x, \alpha)$. Hence, it is proved that $\bar{P}_{d}^{*}(\gamma, \alpha)$ is the smallest concave function greater than or equal to $P_{d}(\gamma, \alpha)$. In addition, since $\bar{P}_{d}^{*}(\gamma, \alpha)$ is monotone increasing (due to the monotone increasing nature of $P_{d}(\gamma, \alpha)$ ), the optimal value of (11a) is equal to $\bar{P}_{d}^{*}\left(\Gamma_{\text {avg }}, \alpha\right)$, which can be achieved by the strategies specified by $(i)$ or $(i i)$ depending on the value of $\Gamma_{\mathrm{avg}}$. The proof for case $(i i i)$, i.e., $\Gamma_{\text {peak }}<\gamma_{t}$, can be obtained in a similar fashion.

Proposition 3 states that when $\alpha<e^{-2}$, time sharing becomes beneficial if the average power limit (equivalently, the average SNR limit) is lower than a certain threshold. In that case, on-off signaling is the optimal strategy, and the duration of the silent period and the transmitted power level are determined according to the average and peak power limits.

Remark: The power allocation strategy can be implemented in practice as follows: Suppose that the statistical model in (1) is valid for $N_{s}$ consecutive transmissions (observations). First, $\gamma_{t}$ defined in Lemma 4 is calculated. Then, if the condition in Proposition 3- $(i)$ is satisfied, the same power level (corresponding to SNR $\Gamma_{\text {avg }}$ ) is used for all $\left(N_{s}\right)$ transmissions. If the condition in Proposition 3-(ii) is satisfied, round $\left(N_{s} \Gamma_{\text {avg }} / \gamma_{t}\right)$ out of $N_{s}$ transmissions occur with a constant power level corresponding to $\operatorname{SNR} \gamma_{t}$, and nothing is transmitted during the remaining slots (corresponding to zero power). A similar approach is adopted if the condition in Proposition 3-(iii) holds. 


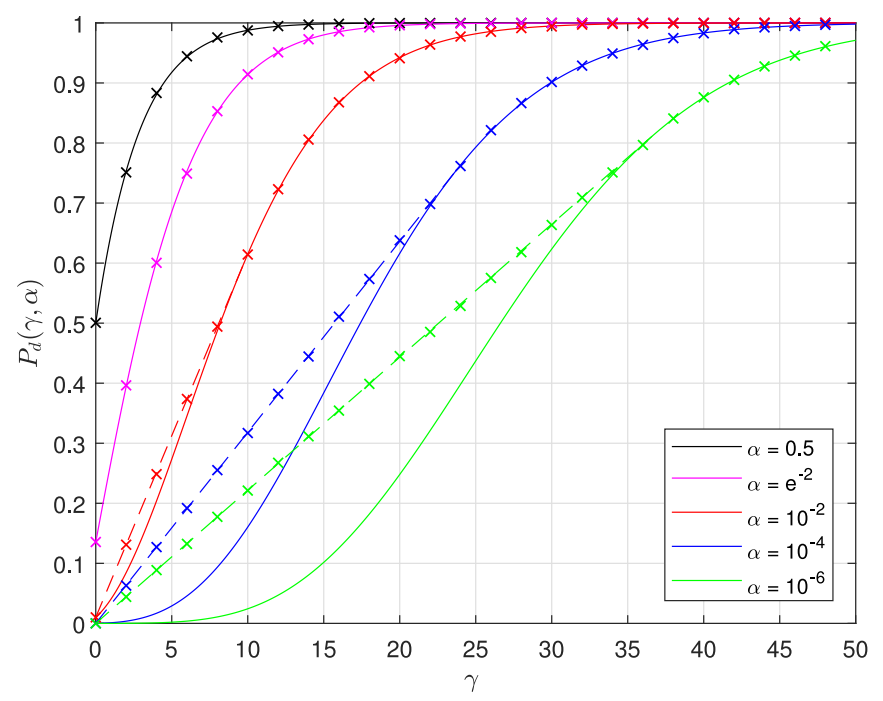

Fig. 1. Probability of detection versus $\gamma$ for various values of the false alarm probability $\alpha$. The dashed lines correspond to the upper boundaries of the convex hulls of $P_{d}(\gamma, \alpha)$ curves, which are attained via on-off signaling, as stated in Proposition 3. The cross signs indicate the results of the Monte-Carlo simulations

\section{NUMERICAL EXAMPLES AND SimULATIONS}

In this section, we provide numerical examples and simulations to illustrate the theoretical results of the previous section. Fig. 1 shows the probability of detection in (5) versus SNR, $\gamma$, for various values of the false alarm probability $\alpha$. The cross $(\times)$ signs in the figure indicate the results of the Monte-Carlo simulations, which match perfectly with the theoretical results (dashed and straight lines), as expected. As stated in Propositions 1 and 2, the probability of detection is a concave function of SNR for $\alpha>e^{-2} \approx 0.135$, and initially a convex and then a concave function of SNR for $\alpha<e^{-2}$. The optimal power allocation strategies can also be deduced from Fig. 1 as follows: Suppose that $\Gamma_{\text {peak }}=50$. Then, the optimal strategy is to operate at the average power limit for $\alpha=0.5$ and $\alpha=e^{-2}$ due to the concavity of the probability of detection. On the other hand, for $\alpha=10^{-2}, \alpha=10^{-4}$, and $\alpha=10^{-6}$, the optimal strategy is to time share between SNRs of 0 and $\gamma_{t}$, with fraction of time $\Gamma_{\mathrm{avg}} / \gamma_{t}$ allocated to the SNR of $\gamma_{t}$ (see Proposition 3), where $\gamma_{t}$ is equal to 9.685, 23.76, and 36.6 for $\alpha=10^{-2}, \alpha=10^{-4}$, and $\alpha=10^{-6}$, respectively. For example, for $\alpha=10^{-4}$ and $\Gamma_{\text {avg }}=10$, the probability of detection can be improved from 0.161 to 0.318 via time sharing between SNRs of 0 and 23.76. The dashed lines in Fig. 1 indicate the probability of detection values that can be achieved via time sharing (on-off signaling) in the considered scenario. It is noted that time sharing becomes more crucial for low levels of false alarm probability, which is the case in many practical scenarios.

\section{EXTENSION TO FADING CHANNELS}

Although no fading is considered in the analysis in Section III, the results are also valid for frequency-flat block-fading channels assuming that perfect channel power gain information is available at the transmitter and peak/average power constraints are imposed over the duration of block-fading. In particular, considering the following observation model

$$
\mathcal{H}_{0}: Y_{k}=N_{k}, \mathcal{H}_{1}: Y_{k}=\sqrt{P h} s_{k}(\theta)+N_{k}, \text { for } k=1, \ldots, n
$$

where $h>0$ is the channel power gain, the only modification in the formulations would be to scale $\operatorname{SNR}(\gamma)$ with the known channel power gain $h$. Under the block-fading channel model, the proposed optimal power allocation approach can be employed within each block. If the transmitter does not have perfect channel power gain information, then the detection probability achieved by the proposed optimal signaling method based on perfect information can be regarded as an upper bound on the detection performance.

If power allocation is applied over different fading blocks, then the convexity properties of the average detection probability should be considered to determine the optimal power allocation strategy. It is noted that for a given value of $h$ in (13), the size- $\alpha$ NP decision rule in (4) is still optimal since the detector threshold does not depend on $P$ or $h$. By defining $\gamma \triangleq n \overline{a^{2}} P h /\left(2 \sigma^{2}\right)$, it is seen that the detection probability of the optimum size- $\alpha$ NP detector for fixed channel power gain $h$ is in the same form as that given in (5). By treating the channel power gain $h$ as a random variable, the detection probability can be averaged over the distribution of $h$ (or, equivalently $\gamma$ ). Since the resulting average detection probability is a function of the transmit power $P$, its convexity properties w.r.t. $P$ can be identified and the optimal power allocation under peak and average power constraints can be determined. To this end, we compute the average detection probability of the proposed detector under Rayleigh block-fading in the following.

For the Rayleigh fading scenario, the probability density function (PDF) of $h$ is given by $f_{h}(h)=(1 / \bar{h}) e^{-h / \bar{h}}$ for $h \geq 0$. For convenience, define $\rho \triangleq n \overline{a^{2}} P /\left(2 \sigma^{2}\right)$; then $\gamma=\rho h$ and $\bar{\gamma}=E_{h}[\gamma]=\rho \bar{h}$, where $E_{h}[\cdot]$ represents expectation w.r.t. fading power distribution. Denote the average detection probability under Rayleigh fading as $\overline{P_{d}}(\bar{\gamma}, \alpha)$. Then, from (5) and [11, Eq. (30)], $\overline{P_{d}}(\bar{\gamma}, \alpha)$ can be calculated as follows:

$$
\begin{aligned}
\overline{P_{d}}(\bar{\gamma}, \alpha) & =\int_{0}^{\infty} \frac{1}{\bar{h}} e^{-\frac{h}{\bar{h}}} Q_{1}[\sqrt{\rho h}, \sqrt{-2 \log \alpha}] d h \\
& =\int_{0}^{\infty} \frac{1}{\bar{h}} e^{-\frac{u^{2}}{\bar{h}}} Q_{1}[u \sqrt{\rho}, \sqrt{-2 \log \alpha}] 2 u d u \\
& =\alpha^{\frac{1}{1+\rho h / 2}}=\alpha^{\frac{1}{1+\bar{\gamma} / 2}} .
\end{aligned}
$$

The second derivative of the average detection probability with respect to the average SNR at the receiver, denoted by ${\overline{P_{d}}}^{\prime \prime}(\bar{\gamma}, \alpha)$, can be computed as

$$
{\overline{P_{d}}}^{\prime \prime}(\bar{\gamma}, \alpha)=\alpha^{\frac{1}{1+\bar{\gamma} / 2}} \frac{1}{2(1+\bar{\gamma} / 2)^{3}} \ln (\alpha)\left(\frac{\ln (\alpha)}{2(1+\bar{\gamma} / 2)}+1\right)
$$

Since $0<\alpha<1$, it is noted that

$$
{\overline{P_{d}}}^{\prime \prime}(\bar{\gamma}, \alpha)>0 \Longleftrightarrow \bar{\gamma}<-\ln (\alpha)-2
$$

Therefore, it is concluded that if $\alpha>e^{-2}$, the average probability of detection is always concave with respect to $\bar{\gamma}$. Otherwise $\overline{P_{d}}(\bar{\gamma}, \alpha)$ is a strictly convex function of $\bar{\gamma}$ for $\bar{\gamma}<-\ln (\alpha)-2$ and a strictly concave function of $\bar{\gamma}$ for $\bar{\gamma}>-\ln (\alpha)-2$. Due to the similarity of 
the convexity properties of the average detection probability to those of the non-fading scenario in Section III-A, the power allocation approach in Section III-B can also be employed for Rayleigh block-fading channels.

\section{CONCLUDING REMARKS}

In this paper, for optimal noncoherent detection of a modulated sinusoidal carrier, the convexity properties of the detection probability have been characterized with respect to the SNR for all values of the false alarm probability. Since required levels of false alarm probability are lower than $e^{-2} \approx 0.135$ in almost all practical applications, time sharing in the form of on-off signaling may prove useful for enhancing the noncoherent detection performance of a modulated sinusoidal carrier.

An important direction for future work is to characterize the convexity properties of the detection probability for fast fading channels.

\section{APPENDIX}

\section{A. Proof of Lemma 1}

Since the second derivative of $P_{d}(\gamma, \alpha)$ is continuous with respect to $\gamma$, the statement in the lemma can be proved by showing that

$$
\left.\lim _{z \downarrow 0} \frac{\partial^{2} P_{d}(\gamma, \alpha)}{\partial \gamma^{2}}\right|_{\gamma=z}>0
$$

for $\alpha \in\left(0, e^{-2}\right)$. In other words, the condition in (17) guarantees that there exists $\hat{\gamma}>0$ such that $P_{d}(\gamma, \alpha)$ is convex in $[0, \hat{\gamma}]$. Towards the aim of proving (17), the second derivative of $P_{d}(\gamma, \alpha)$ with respect to $\gamma$ is obtained as follows:

$$
\begin{aligned}
\frac{\partial^{2} P_{d}(\gamma, \alpha)}{\partial \gamma^{2}}= & e^{-\frac{\gamma}{2}}\left(\frac{1}{4} \int_{f(\alpha)}^{\infty} x e^{-\frac{x^{2}}{2}} I_{0}(\sqrt{\gamma} x) d x\right. \\
& -\int_{f(\alpha)}^{\infty} x e^{-\frac{x^{2}}{2}} g(x, \gamma) d x \\
& \left.+\int_{f(\alpha)}^{\infty} x e^{-\frac{x^{2}}{2}} h(x, \gamma) d x\right),
\end{aligned}
$$

where $g(x, \gamma)=\frac{x^{2}}{4 \pi} \int_{0}^{2 \pi} \sin ^{2} \theta e^{x \sqrt{\gamma} \cos \theta} d \theta \quad$ and $\quad h(x, \gamma)=\frac{x^{4}}{24 \pi}$ $\int_{0}^{2 \pi} \sin ^{4} \theta e^{x \sqrt{\gamma} \cos \theta} d \theta$. Then, the following three results are utilized in the proof.

$$
\begin{aligned}
& \lim _{\gamma \downarrow 0} e^{-\frac{\gamma}{2}} \int_{f(\alpha)}^{\infty} \frac{1}{4} x e^{-\frac{x^{2}}{2}} I_{0}(\sqrt{\gamma} x) d x=\int_{f(\alpha)}^{\infty} \frac{1}{4} x e^{-\frac{x^{2}}{2}} d x, \\
& \lim _{\gamma \downarrow 0} e^{-\frac{\gamma}{2}} \int_{f(\alpha)}^{\infty} x e^{-\frac{x^{2}}{2}} g(x, \gamma) d x=\int_{f(\alpha)}^{\infty} \frac{1}{4} x^{3} e^{-\frac{x^{2}}{2}} d x, \\
& \lim _{\gamma \downarrow 0} e^{-\frac{\gamma}{2}} \int_{f(\alpha)}^{\infty} x e^{-\frac{x^{2}}{2}} h(x, \gamma) d x=\int_{f(\alpha)}^{\infty} \frac{1}{32} x^{5} e^{-\frac{x^{2}}{2}} d x .
\end{aligned}
$$

Here, the proof for (19) is provided ((20) and (21) can be shown in a similar fashion). Notice that from the monotonicity of $I_{0}(\cdot)$ for $\gamma \in[0,1]$, it follows that $e^{-\frac{\gamma}{2}} \frac{1}{4} x e^{-\frac{x^{2}}{2}} I_{0}(\sqrt{\gamma} x) \leq x e^{-\frac{x^{2}}{2}} I_{0}(x)$. Since $x e^{-\frac{x^{2}}{2}} I_{0}(x)$ is integrable, by the Dominated Convergence Theorem, the expression on the left-hand-side (LHS) of (19) can be written as

$$
\begin{aligned}
& \lim _{\gamma \downarrow 0} \int_{f(\alpha)}^{\infty} \frac{e^{-\frac{\gamma}{2}}}{4} x e^{-\frac{x^{2}}{2}} I_{0}(\sqrt{\gamma} x) d x \\
= & \int_{f(\alpha)}^{\infty} \lim _{\gamma \downarrow 0} \frac{e^{-\frac{\gamma}{2}}}{4} x e^{-\frac{x^{2}}{2}} I_{0}(\sqrt{\gamma} x) d x
\end{aligned}
$$

Since $\lim _{\gamma \downarrow 0} \frac{e^{-\frac{\gamma}{2}}}{4} x e^{-\frac{x^{2}}{2}} I_{0}(\sqrt{\gamma} x)=\frac{1}{4} x e^{-\frac{x^{2}}{2}}$, the statement in (19) is proved. In a similar manner, it can be shown that $\lim _{\gamma \downarrow 0} g(x, \gamma)=x^{2} / 4$ and $\lim _{\gamma \downarrow 0} h(x, \gamma)=x^{4} / 32$.

By combining the results in (19)-(21) with (18), it is seen that

$$
\begin{aligned}
\left.\lim _{z \downarrow 0} \frac{\partial^{2} P_{d}(\gamma, \alpha)}{\partial \gamma^{2}}\right|_{\gamma=z}= & \frac{1}{4}\left(\int_{f(\alpha)}^{\infty} \frac{1}{8} x^{5} e^{-\frac{x^{2}}{2}} d x\right. \\
& \left.-\int_{f(\alpha)}^{\infty} x^{3} e^{-\frac{x^{2}}{2}} d x+\int_{f(\alpha)}^{\infty} x e^{-\frac{x^{2}}{2}} d x\right) .
\end{aligned}
$$

Then, it is obtained that

$$
\left.\lim _{z \downarrow 0} \frac{\partial^{2} P_{d}(\gamma, \alpha)}{\partial \gamma^{2}}\right|_{\gamma=z}=\frac{1}{8} f(\alpha)^{2} e^{\frac{-f(\alpha)^{2}}{2}}\left(\frac{f(\alpha)^{2}}{4}-1\right) .
$$

Thus, the expression on the LHS of (24) is positive if and only if $f(\alpha)^{2}>4$, which is satisfied if and only if $\alpha<e^{-2}$.

\section{B. Proof of Lemma 2}

Similar to the proof of Proposition 1 (see (7)), we consider the sign of

$$
\frac{f(\alpha)}{\sqrt{\gamma}} I_{2}(\sqrt{\gamma} f(\alpha))-I_{1}(\sqrt{\gamma} f(\alpha)) .
$$

This sign determines the convexity/concavity of the detection probability. From [12, Cor. 1], it can be seen that $I_{2}(\sqrt{\gamma} f(\alpha))<I_{1}(\sqrt{\gamma} f(\alpha)) e^{-\alpha_{0} \frac{3}{2 \sqrt{\gamma} f(\alpha)}}$ for $\sqrt{\gamma} f(\alpha) \geq 2$, where $\alpha_{0}=-\log (\sqrt{2}-1)$. Then, as $\alpha_{0}>0$, it is clear that $I_{2}(\sqrt{\gamma} f(\alpha))<$ $I_{1}(\sqrt{\gamma} f(\alpha))$ for $\sqrt{\gamma} f(\alpha) \geq 2$. Therefore, the statement in the lemma follows directly for $\gamma \geq \max \left\{(f(\alpha))^{2}, 2 /(f(\alpha))^{2}\right\}$. Namely, it is sufficient to choose $\tilde{\gamma}=\max \left\{(f(\alpha))^{2}, 2 /(f(\alpha))^{2}\right\}$ for a fixed $\alpha$.

\section{Proof of Lemma 3}

From (7), notice that if $P_{d}^{\prime \prime}\left(\gamma^{*}, \alpha\right)=0$ for $\gamma^{*}<\infty$, then $\gamma^{*}$ must be a root of $\frac{f(\alpha)}{\sqrt{\gamma}} I_{2}(\sqrt{\gamma} f(\alpha))-I_{1}(\sqrt{\gamma} f(\alpha))$. Now observe that

$$
\begin{aligned}
& \frac{f(\alpha)}{\sqrt{\gamma}} I_{2}(\sqrt{\gamma} f(\alpha))-I_{1}(\sqrt{\gamma} f(\alpha)) \\
& =I_{1}(\sqrt{\gamma} f(\alpha))\left(\frac{f(\alpha)}{\sqrt{\gamma}} \frac{I_{2}(\sqrt{\gamma} f(\alpha))}{I_{1}(\sqrt{\gamma} f(\alpha))}-1\right) .
\end{aligned}
$$

Since $I_{1}(\cdot)>0, \gamma^{*}$ must be a root of $\frac{f(\alpha)}{\sqrt{\gamma}} \frac{I_{2}(\sqrt{\gamma} f(\alpha))}{I_{1}(\sqrt{\gamma} f(\alpha))}-1$, which can be expressed as

$$
\frac{f(\alpha)}{\sqrt{\gamma}} \frac{I_{2}(\sqrt{\gamma} f(\alpha))}{I_{1}(\sqrt{\gamma} f(\alpha))}-1=f(\alpha)^{2}\left(\frac{1}{v_{1}(\sqrt{\gamma} f(\alpha))}-\frac{1}{f(\alpha)^{2}}\right),
$$

where $v_{1}(x) \triangleq x I_{1}(x) / I_{2}(x)$ As stated in [9] and [13], $v_{1}(x)$ is a strictly increasing function for positive $x$. Therefore, in our case, 
$\frac{1}{v_{1}(\sqrt{\gamma} f(\alpha))}$ is a strictly decreasing function of $\gamma$, which implies that there must be at most one root of (27); hence, there is at most one finite root of $P_{d}^{\prime \prime}(\gamma, \alpha)$. Based on Lemma 1 and 2, there is at least one finite root of $P_{d}^{\prime \prime}(\gamma, \alpha)$ when $\alpha<e^{-2}$ by the Intermediate Value Theorem. Therefore, there exists a unique inflection point.

\section{Proof of Lemma 4}

To prove Lemma 4, the following result is obtained first.

Lemma 5: $\lim _{\gamma \rightarrow \infty} P_{d}(\gamma, \alpha)=1$.

Proof: From [14, Eq. (4)], the detection probability can be lower bounded for $\sqrt{\gamma} \geq f(\alpha)$ as follows:

$$
Q_{1}[\sqrt{\gamma}, f(\alpha)] \geq 1-\frac{1}{2}\left(e^{-\frac{\left.(\sqrt{\gamma}-f(\alpha))^{2}\right)}{2}}-e^{-\frac{\left.(\sqrt{\gamma}+f(\alpha))^{2}\right)}{2}}\right)
$$

which can equivalently be written as

$$
Q_{1}[\sqrt{\gamma}, f(\alpha)] \geq 1-\frac{1}{2} e^{-\frac{\gamma}{2}} \alpha\left(e^{\sqrt{\gamma} f(\alpha)}-e^{-\sqrt{\gamma} f(\alpha)}\right) .
$$

For a fixed $\alpha$, the right-hand-side (RHS) of (29) converges to 1 as $\gamma$ goes to $\infty$. Therefore, it is concluded that $\lim _{\gamma \rightarrow \infty} P_{d}(\gamma, \alpha) \geq 1$. Also, as $P_{d}(\gamma, \alpha)$ is the probability of detection, it must be less than or equal to 1 . Hence, the statement in Lemma 5 follows.

Let $\tilde{g}(\gamma)$ denote the straight line passing through points $\left(0, P_{d}(0, \alpha)\right)$ and $\left(\gamma_{t}, P_{d}\left(\gamma_{t}, \alpha\right)\right)$, which has a slope of $P_{d}^{\prime}\left(\gamma_{t}, \alpha\right)$. Then,

$$
\tilde{g}(\gamma)=\tilde{g}(0)+P_{d}^{\prime}\left(\gamma_{t}, \alpha\right) \gamma
$$

where $P_{d}^{\prime}\left(\gamma_{t}, \alpha\right)$ is the first derivative of $P_{d}(\gamma, \alpha)$ with respect to $\gamma$ evaluated at $\gamma_{t}$. By definition, $\tilde{g}(0)=P_{d}(0, \alpha)$. First, it is noted that $P_{d}(0, \alpha)=\int_{f(\alpha)}^{\infty} x e^{-\frac{x^{2}}{2}} I_{0}(0) d x$. Since $I_{0}(0)=1$, $P_{d}(0, \alpha)$ is calculated as $P_{d}(0, \alpha)=e^{\frac{-f(\alpha)^{2}}{2}}=\alpha$. Therefore, the existence of $\gamma_{t}$ such that $P_{d}\left(\gamma_{t}, \alpha\right)=\alpha+P_{d}^{\prime}\left(\gamma_{t}, \alpha\right) \gamma_{t}$ will imply the existence of the straight line. Define a new function as $\tilde{h}(\gamma) \triangleq P_{d}(\gamma, \alpha)-\alpha-P_{d}^{\prime}(\gamma, \alpha) \gamma$. If one can show that there exists $\gamma_{t} \neq 0$ such that $\tilde{h}\left(\gamma_{t}\right)=0$, then the claim will be proved. Notice that $\tilde{h}(0)=0$ and $\tilde{h}^{\prime}(\gamma)=P_{d}^{\prime}(\gamma, \alpha)-P_{d}^{\prime \prime}(\gamma, \alpha) \gamma-P_{d}^{\prime}(\gamma, \alpha)=$ $-P_{d}^{\prime \prime}(\gamma, \alpha) \gamma$. From Proposition $2, \tilde{h}^{\prime}(\gamma)<0$ if $\gamma \in\left[0, \gamma_{\alpha}\right]$ and $\tilde{h}^{\prime}(\gamma)>$ 0 if $\gamma \in\left(\gamma_{\alpha}, \infty\right)$. Therefore, $\tilde{h}$ is a decreasing function in $\left[0, \gamma_{\alpha}\right]$ and an increasing function in $\left(\gamma_{\alpha}, \infty\right)$. Hence, it is sufficient to show that $\lim _{\gamma \rightarrow \infty} \tilde{h}(\gamma)>0$ since this dictates the existence of such a $\gamma_{t}$ due to the Intermediate Value Theorem.

From Lemma 5, the following relation is obtained:

$$
\begin{aligned}
\lim _{\gamma \rightarrow \infty} \tilde{h}(\gamma) & =\lim _{\gamma \rightarrow \infty} P_{d}(\gamma, \alpha)-\alpha-P_{d}^{\prime}(\gamma, \alpha) \gamma \\
& =1-\alpha-\lim _{\gamma \rightarrow \infty} P_{d}^{\prime}(\gamma, \alpha) \gamma
\end{aligned}
$$

Therefore, if we can show that $\lim _{\gamma \rightarrow \infty} P_{d}^{\prime}(\gamma, \alpha) \gamma<1-\alpha$, then $\lim _{\gamma \rightarrow \infty} \tilde{h}(\gamma)>0$ will be proved. Notice that $\left(P_{d}(\gamma, \alpha)-1\right)$ goes to 0 and $\frac{1}{\gamma}$ goes to 0 as $\gamma$ goes to $\infty$. Then, by L'Hôpital Rule, the following expressions are derived:

$$
\begin{aligned}
\lim _{\gamma \rightarrow \infty}\left(P_{d}(\gamma, \alpha)-1\right) \gamma & =\lim _{\gamma \rightarrow \infty} \frac{P_{d}(\gamma, \alpha)-1}{1 / \gamma} \\
& =\lim _{\gamma \rightarrow \infty} \frac{P_{d}^{\prime}(\gamma, \alpha)}{-1 / \gamma^{2}}=\lim _{\gamma \rightarrow \infty}-P_{d}^{\prime}(\gamma, \alpha) \gamma^{2}
\end{aligned}
$$

Therefore, it can be deduced that $\lim _{\gamma \rightarrow \infty}\left(P_{d}(\gamma, \alpha)-1\right) \gamma=0$ if and only if $\lim _{\gamma \rightarrow \infty} P_{d}^{\prime}(\gamma, \alpha) \gamma^{2}=0$. Since $0 \leq\left|P_{d}^{\prime}(\gamma, \alpha) \gamma\right| \leq$ $\left|P_{d}^{\prime}(\gamma, \alpha) \gamma^{2}\right|$ for $\gamma \geq 1, \quad \lim _{\gamma \rightarrow \infty} P_{d}^{\prime}(\gamma, \alpha) \gamma^{2}=0$ implies that $\lim _{\gamma \rightarrow \infty} P_{d}^{\prime}(\gamma, \alpha) \gamma=0$. Hence, proving that $\lim _{\gamma \rightarrow \infty}\left(P_{d}(\gamma, \alpha)-\right.$ 1) $\gamma=0$ would be sufficient to conclude that $\lim _{\gamma \rightarrow \infty} P_{d}^{\prime}(\gamma, \alpha) \gamma=0$. For this reason, we next compute $\lim _{\gamma \rightarrow \infty}\left(P_{d}(\gamma, \alpha)-1\right) \gamma$. As $P_{d}$ is the detection probability, $P_{d}(\gamma, \alpha)-1 \leq 0$; therefore, $\lim _{\gamma \rightarrow \infty}\left(P_{d}(\gamma, \alpha)-1\right) \gamma \leq 0$. For the other direction, from [14, Eq. (4)], it is known that for $\sqrt{\gamma} \geq f(\alpha), \quad P_{d}(\gamma, \alpha) \geq 1-$ $\frac{1}{2} e^{-\frac{\gamma}{2}} \alpha\left(e^{\sqrt{\gamma} f(\alpha)}-e^{-\sqrt{\gamma} f(\alpha)}\right)$. Then, for $\sqrt{\gamma} \geq f(\alpha)$

$$
\gamma\left(P_{d}(\gamma, \alpha)-1\right) \geq-\frac{\gamma}{2} e^{-\frac{\gamma}{2}} \alpha\left(e^{\sqrt{\gamma} f(\alpha)}-e^{-\sqrt{\gamma} f(\alpha)}\right) .
$$

For a fixed $\alpha$, the RHS of (35) converges to 0 . Therefore, $\lim _{\gamma \rightarrow \infty}\left(P_{d}(\gamma, \alpha)-1\right) \gamma \geq 0$. Hence, the converse direction is shown. Overall, it is obtained that $\lim _{\gamma \rightarrow \infty}\left(P_{d}(\gamma, \alpha)-1\right) \gamma=0$. This implies that $\lim _{\gamma \rightarrow \infty} \tilde{h}(\gamma)=1-\alpha>0$ as $\alpha<e^{-2}$, which concludes the proof.

\section{REFERENCES}

[1] S. M. Elnoubi, "Probability of error analysis of digital partial response continuous phase modulation with noncoherent detection in mobile radio channels," IEEE Trans. Veh. Technol., vol. 38, no. 1, pp. 19-30, Feb. 1989.

[2] H. V. Poor, An Introduction to Signal Detection and Estimation, 2nd ed New York, NY, USA: Springer-Verlag, 1994.

[3] M. Azizoglu, "Convexity properties in binary detection problems," IEEE Trans. Inf. Theory, vol. 42, no. 4, pp. 1316-1321, Jul. 1996.

[4] S. Loyka, V. Kostina, and F. Gagnon, "Symbol error rates of maximum-likelihood detector: Convex/concave behavior and applications," in Proc. IEEE Int. Symp. Inf. Theory, Jun. 2007, pp. 2501-2505.

[5] B. Dulek, S. Gezici, and O. Arikan, "Convexity properties of detection probability under additive Gaussian noise: Optimal signaling and jamming strategies," IEEE Trans. Signal Process., vol. 61, no. 13, pp. 3303-3310, Jul. 2013.

[6] Y. Sun, A. Baricz, and S. Zhou, "On the monotonicity, log-concavity and tight bounds of the generalized Marcum and Nuttall Q-functions," IEEE Trans. Inf. Theory, vol. 56, no. 3, pp. 1166-1186, Mar. 2010.

[7] Y. A. Brychkov, "On some properties of the Marcum Q function," Integral Transforms Special Functions, vol. 23, no. 3, pp. 177-182, 2012.

[8] E. K. Ifantis and P. D. Siafarikas, "Inequalities involving Bessel and modified Bessel functions," J. Math. Anal. Appl., vol. 147, no. 1, pp. 214-227, 1990.

[9] A. Baricz, "Tight bounds for the generalized Marcum Q-function," J. Math. Anal. Appl., vol. 360, no. 1, pp. 265-277, Dec. 2009.

[10] H. Chen, P. K. Varshney, S. M. Kay, and J. H. Michels, "Theory of the stochastic resonance effect in signal detection: Part I-Fixed detectors," IEEE Trans. Signal Process., vol. 55, no. 7, pp. 3172-3184, Jul. 2007.

[11] A. H. Nuttall, "Some integrals involving the $q_{m}$-function," Naval Underwater Syst. Center, New London, CT, USA, Tech. Rep. AD-779 846, May 1974.

[12] P. Balachandran, W. Viles, and E. D. Kolaczyk, "Exponential-type inequalities involving ratios of the modified Bessel function of the first kind and their applications," unpublished paper, 2013. [Online]. Available: https://arxiv.org/abs/1311.1450

[13] H. C. Simpson, and S. J. Spector, "Some monotonicity results for ratios of modified Bessel functions," Quart. Appl. Math., vol. 42, no. 1, pp. 95-98, Apr. 1984.

[14] M. K. Simon and M. S. Alouini, "Exponential-type bounds on the generalized Marcum Q-function with application to error probability analysis over fading channels," IEEE Trans. Commun., vol. 48, no. 3, pp. 359-366, Mar. 2000. 